\title{
Robustness of ultrasonic detection of flaws by using synthetic aperture focusing technique
}

\author{
Sofia Rejani ${ }^{1, a}$, Abdellatif Khamlichi ${ }^{2}$ and Abdellah El-Hajjaji ${ }^{1}$ \\ ${ }^{1}$ SCD Laboratory, Faculty of Sciences, University Abdelmalek Essaadi, Tetouan 93030, Morocco \\ ${ }^{2}$ National School of Applied Sciences at Tetouan, University Abdelmalek Essaadi, Tetouan 93030, Morocco
}

\begin{abstract}
One of the challenging problems in non-destructive evaluation is related to identification and sizing of flaws. A high resolution image of the scanned part is required. This allows, through using adequate post-processing of data, to perform localisation and sizing of a flaw. Several techniques have been introduced recently for this purpose. These include among others the synthetic aperture focusing technique, inverse wave-field extrapolation and the total focusing method. However, large uncertainties are affecting the inverse problem solution as provided by these methods when dealing with small defects. It was recognized that reconstruction based on the ultrasonic synthetic aperture focusing technique elaborated in frequency domain provides high resolution imaging even at large distances. This work focused on this promising procedure for the special case of ultrasonic imaging of flaws in 2D elastic medium under plane strain conditions, where the image is provided by a B-scan. Robustness of detection was investigated through perturbing the radargram by white noise and assessed as function of noise energy. It was found that synthetic aperture focusing technique is insensitive to noise.
\end{abstract}

\section{Introduction}

Flaws in parts can be produced during the manufacturing process or in service due to fatigue or induced accidental damage. In practice, it is important to determine the position of a flaw and estimate its size. Ultrasonic testing can be used for this purpose [1-2]. This may be performed by means of ultrasonic imaging which involves converting raw ultrasonic data collected by a transducer during a B-scan testing into an image representing with sufficient precision the interior state of the examined structure. This conversion can be achieved by the synthetic aperture focusing technique [3].

The concept of synthetic aperture appeared at first in the field of radar in 1951 when the technique of synthetic aperture radar (SAR) imaging was introduced [4]. This approach is based on a combination of measurements taken by moving radar to synthesize electromagnetic signals with a better resolution than that provided by fixed radar. The earlier algorithms dealing with synthetic aperture imaging were developed for SAR applications which are characterized by narrowband transmissions and very high propagation speeds [5]. Later on, the concept of synthetic aperture was proven to be relevant for side-looking sonar [6]. Then, the technique was applied in the field of medical imaging. In the 1970s, the synthetic aperture focusing reached the field of ultrasonics and his name became aperture focusing technique (SAFT) [7]. This flexible imaging method was firstly explored for non-destructive testing using ultrasonic transducers to inspect an isotropic medium. SAFT algorithm was used after that for reconstruction of defects in anisotropic media [8-9] where wave propagation is more complex because of the directional dependence of the material properties.

The SAFT method has been presented with both time domain and frequency domain versions. The frequency based version uses the Fast Fourier Transform (FFT) implementation, while the standard scanning ultrasonic testing has focused on the time domain. A special algorithm called delay and sum (DAS) was devised for this purpose [10]. This algorithm allowed obtaining an image with high resolution independently of the depth when compared with an ordinary B-scan imaging [11]. However, Olofsson has demonstrated the superiority of SAFT in frequency domain by using phased shift migration (PSM) technique [12]. Considering two experiments for immersed isotropic and layered objects in water, he concluded that the PSM is more efficient than the DAS algorithm. Moreover, the PSM algorithm has a lower computational complexity when compared with DAS algorithm. Advantage of the imaging by PSM has been also demonstrated in case of layered medium.

In this work, the SAFT in frequency domain, in its PSM version, will be applied for detection of flaws in a 2D isotropic elastic medium undergoing plane strains deformation. Simulation of B-scan testing is performed by using the Finite Element Method (FEM) under Abaqus software. Perturbation of the radargram is considered through introducing additive white noise in

\footnotetext{
$\overline{{ }^{a} \text { Corresponding author: Sofia.rejiani } @ \text { gmail.com }}$
} 
the signal. Robustness of flaw detection is then investigated as function of the noise energy.

\section{Materials and methods}

The PSM method was first developed by Gazdag in 1978 for seismic records in inhomogeneous media [15]. Then, it was later adapted for ultrasonic imaging. This method is based on the exploding reflector model in which the point scatterers in the medium acts like a source emitting a pulse at $t=0$, and creating a wave field which is recorded by a transducer array. The exploding reflector model enables to consider the point scatterers in medium like point reflectors of the wave field. Besides, it neglects multiple reflections between layers and considers only upward-travelling waves.

\subsection{Algorithm formulation}

Let us consider an acoustic wave propagating in a multilayered medium which geometry is composed by $\mathrm{L}$ layers, numbered $(1, \ldots, \mathrm{L})$ in figure 1 . Each layer $\ell$ has two characteristics: the thickness of the layer, denoted $\mathrm{d}_{\ell}$, and the wave velocity, denoted $\mathrm{c}_{\ell}$. The depth $\mathrm{z}_{\ell}$ represents the depth of the lower side of each layer $\ell$.

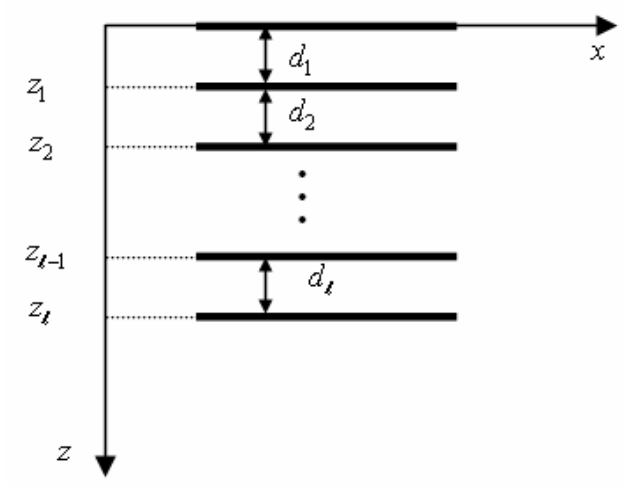

Figure 1. The multilayered geometry of the propagating medium

Let us denote by $p(x, z, t)$ the wave field emitted by the reflectors at $t=0$, and recorded by the transducer array in line $z=0$, this wave field can be extrapolated from the top line to others depths by decomposing it into upward-traveling waves of each layer of the medium and by superimposing it in order to find the final result. This emphasise the main idea encompassed in PSM method which intends extrapolating the observed wave field to create an image of the medium.

The expression of the upward-traveling wave at depth $\ell$ can be written as

$$
p_{\ell}(x, z, t)=P_{\ell}\left(k_{x}, \omega\right) \exp \left[i\left(k_{x} x+k_{z, \ell}\left(z-z_{\ell-1}\right)-\omega t\right)\right]
$$

where $P_{\ell}\left(k_{x}, \omega\right)$ is the complex amplitude, $\omega$ the angular frequency, $k_{x}$ and $k_{z, \ell}$ are the components of the wave number vector along the $x$-axis and $z$-axis .
Using the dispersion relationship

$$
k_{x}^{2}+k_{z, \ell}^{2}=\frac{\omega^{2}}{c_{\ell}^{2}}
$$

one obtains $k_{z, \ell}$ as a function of $k_{x}$ and $c_{\ell}$

$$
k_{z, \ell}=-\sqrt{\frac{\omega^{2}}{c_{\ell}^{2}}-k_{x}^{2}}
$$

with $\frac{\omega^{2}}{c_{\ell}^{2}} \geq k_{x}^{2}$ and the component $k_{z, \ell}<0$ because the wave travels upward along the $z$-axis.

Equation (1) can be rewritten as follows

$$
p_{\ell}(x, z, t)=P_{\ell}\left(k_{x}, \omega, z\right) \exp \left[i\left(k_{x} x-\omega t\right)\right]
$$

with

$$
P_{\ell}\left(k_{x}, \omega, z\right)=P_{\ell}\left(k_{x}, \omega\right) \exp \left[i k_{z, \ell}\left(z-z_{\ell-1}\right)\right]
$$

Equation (4) represents the wave field emitted by the reflectors from the layer $\ell-1$ to the layer $\ell$. Equation (5) gives the expression of the extrapolated wave field from layer $\ell-1$ to layer $\ell$. We can generalize this equation to give the expression of the extrapolated wave field from the top layer 1 to any other layer $\ell$ in the medium, we obtain:

$$
P\left(k_{x}, \omega\right)=P_{\ell}\left(k_{x}, \omega\right) \prod_{n=1}^{\ell-1} \exp \left(i k_{z, n+1} d_{n}\right)
$$

One should notice that equation (4) represents the expression of a two-dimensional Fourier transform on $x$ and $t$, which allows to pass from the temporal domain $p_{\ell}(x, z, t)$ to frequency domain $P_{\ell}\left(k_{x}, \omega, z\right)$. In equation (6) appears the phase shift which is represented by multiplication with the exponential involving the phase shift of all the layers above a given point at depth $z$. From equations (4) and (6), the phase shift migration PSM takes its name.

Considering the wave signal at $z=0$, one obtains then

$$
p(x, z=0, t)=P\left(k_{x}, \omega\right) \exp \left[i\left(k_{x} x-\omega t\right)\right]
$$

From which the amplitude $P\left(k_{x}, \omega\right)$ can be calculated by performing the two-dimensional Fourier transform on $x$ and $t$. Then, by using equation (6), the amplitudes for a given layer can be calculated as

$$
P_{\ell}\left(k_{x}, \omega\right)=P\left(k_{x}, \omega\right) \prod_{n=1}^{\ell-1} \exp \left(-i k_{z, n+1} d_{n}\right)
$$

Using now equation (5), the amplitude at any depth $z$ for a given wave frequency is obtained as

$$
P_{\ell}\left(k_{x}, \omega, z\right)=P_{\ell}\left(k_{x}, \omega\right) \exp \left[i k_{z, \ell}\left(z-z_{\ell-1}\right)\right]
$$

By using equation (4) the wave signal at any depth $z$ is

$$
p_{\ell}(x, z, t)=P_{\ell}\left(k_{x}, \omega, z\right) \exp \left[i\left(k_{x} x-\omega t\right)\right]
$$


Since in equation (10), the dependency on the layer is implicit, one can write simply

$$
p(x, z, t)=P\left(k_{x}, \omega, z\right) \exp \left[i\left(k_{x} x-\omega t\right)\right]
$$

To obtain an image of the medium using PSM method, it is necessary to apply integration over $\omega$ in the frequency domain, followed by a 1-D inverse Fourier transform on $x$, at $t=0$ which indicates the time when the pulse is emitted from the reflectors. This corresponds to the time when a maximum concentration of the wave field occurs or in other terms the time that gives a focused image. The focused imaged pixel writes

$$
p(x, z, t=0)=\iint_{-\infty}^{+\infty} P\left(k_{x}, \omega, z\right) \exp \left(i k_{x} x\right) d k_{x} d \omega
$$

\subsection{The imaging procedure by PSM}

In summary, PSM imaging of a medium is done through the following procedure:

1. Calculate the two-dimensional Fourier transform of the radargram as obtained from a B-scan, where the transducer is moved along the $x$-axis for $z=0$ according to equation (7).

2. Calculate the wave field at the top layer interface using equation (8).

3. For each depth $z=z_{\ell}+\Delta z$ within the layer $\ell$, shift the wave field by multiplying it with an exponential using (9).

4. Create a focused image by application of equation (12).

\section{Results and discussion}

\subsection{Delamination detection by using PSM}

Let us consider a plane strain elastic problem on a rectangular domain with the presence of a defect having the form of a cut-out as shown in Figure 2.

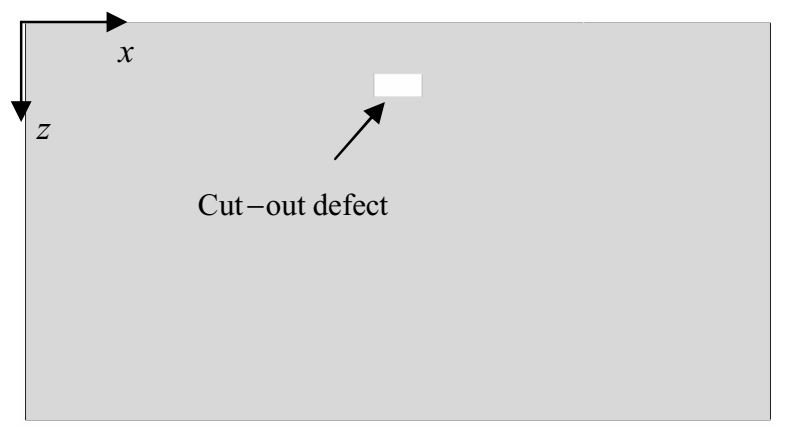

Figure 2. Plane strain elastic domain with a cut-out defect.

The rectangle has dimensions: length $a=300 \mathrm{~mm}$, height $b=160 \mathrm{~mm}$. The cut-out has the form of a rectangle which is centered in $x$-axis direction and located at depth $d=20 \mathrm{~mm}$. The cut-out dimensions are length $a^{\prime}=20 \mathrm{~mm}$ and height $b^{\prime}=10 \mathrm{~mm}$. The defect size is chosen here large enough in order to avoid numerical discrepancies which could result from a very small defect. Here the objective is feasibility of the method with testing its robustness firstly for relatively large defects. This feature should also be tested for smaller flaws, but this issue is not considered in the actual study.

The material properties are density $\rho=2700 \mathrm{~kg} / \mathrm{m}^{3}$, Young modulus $E=70 \mathrm{GPa}$, Poisson coefficient $v=0.25$. These yield for the velocity of compressive waves $c_{p}=5577.7 \mathrm{~m} / \mathrm{s}$ and the shear waves velocity $c_{s}=3220.3 \mathrm{~m} / \mathrm{s}$.

The B-scan was achieved by selecting equally spaced points at uniform interval distance $\Delta x=4.688 \mathrm{~mm}$ between points, beginning from the abscissa $79.687 \mathrm{~mm}$ to the abscissa $220.312 \mathrm{~mm}$, where the origin is selected on the left top corner of the rectangle. A total of 30 points were considered for excitation and recording at the same point the displacement along the $z$-axis direction. The excitation at a given point was a concentrated force having the form of a pulse, applied along the $z$-direction, and with the characteristics depicted in Figure3.

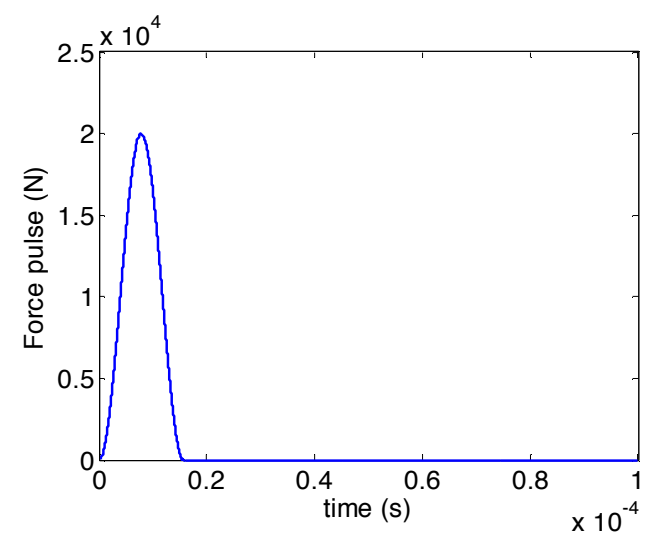

Figure 3. The pulse excitation vertical force

The step time was a user-defined increment of value $\Delta t_{c}=2 n s$, the results are stored at the sampling time increment $\Delta t=1 \mu s$, so the sampling frequency is $f_{S}=1 M H z$. The obtained raw data radargram is shown in Figure 4.

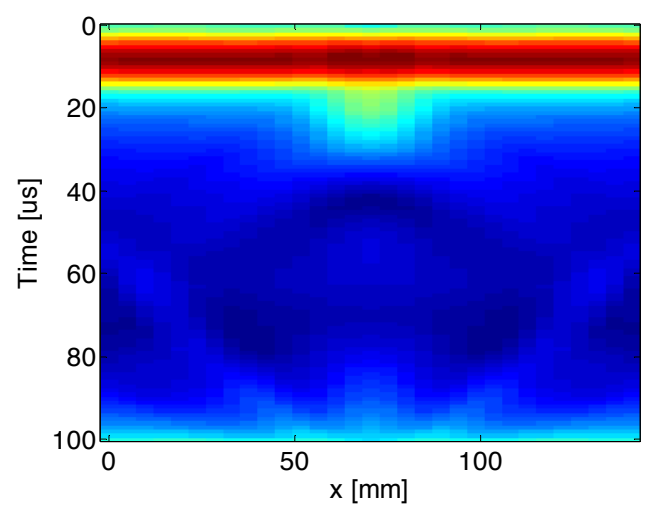

Figure 4. The raw data radargram before application of PSM. 
The PSM algorithm is applied on the raw data in order to reconstruct the image of the rectangular domain with the cut-out defect. The obtained focused image is shown in Figure 5.

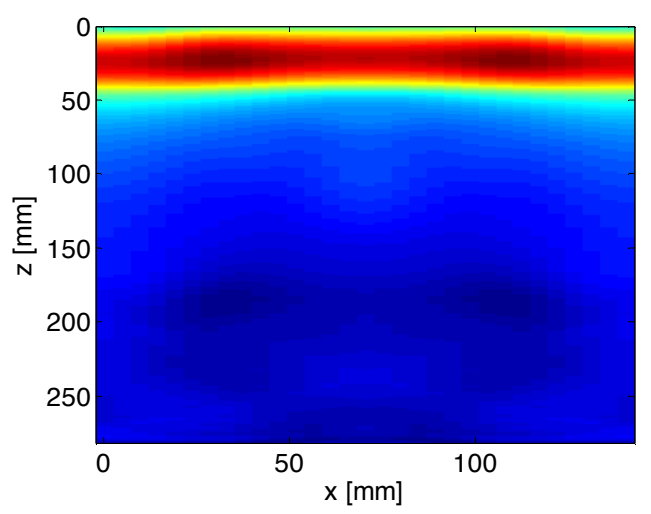

Figure 5. The focused image for literal data.

\subsection{Robustness of detection by PSM algorithm}

To assess robustness of PSM algorithm, the raw data was perturbed with a random noise. This noise affected each term of the raw data matrix, which in fact the tow-way travel time, with a pre-defined level of energy. Two levels of noise amplitude were studied: $20 \%$ and $100 \%$. Figure 6 and Figure 7 presents respectively the focused image for $20 \%$ and $100 \%$ noise level.

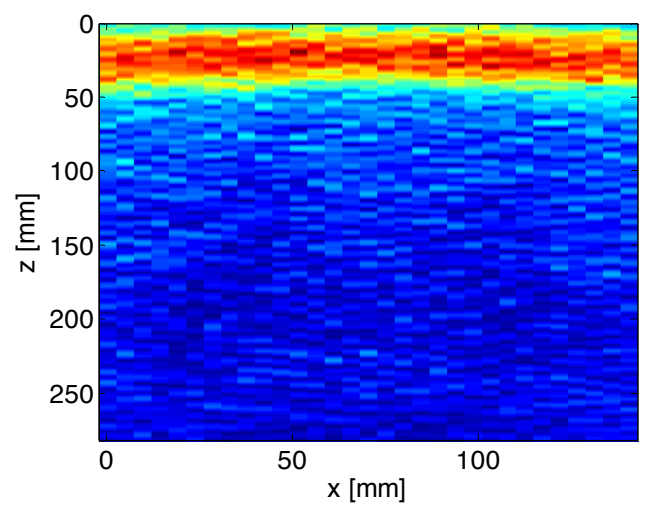

Figure 6. The focused image with $20 \%$ of noise influence.

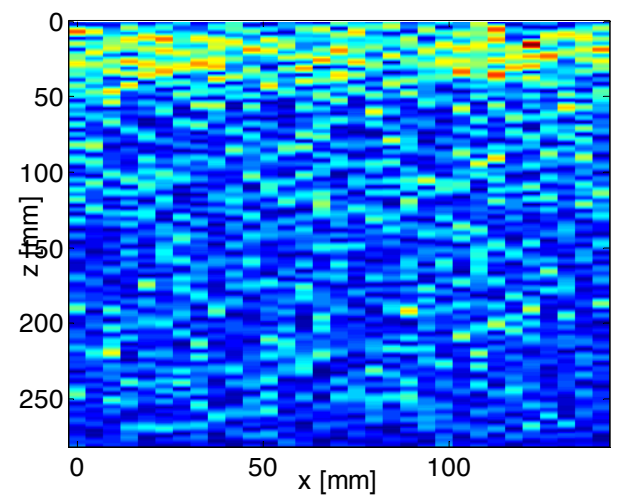

Figure 7. The focused image with $100 \%$ of noise influence.
From Figure 6, one can conclude that the algorithm is very robust in detecting defects as the defect signature is still clearly visible even for perturbations that have amplitudes reaching $20 \%$ of that of the acquired signal.

\section{Conclusions}

The synthetic aperture focusing technique was considered in this work in order to operate detection of flaws in two-dimensional elastic medium under plane stress deformation. Large uncertainties have been assumed to affect the raw data radargram acquired according to a B-scan. It was found that the synthetic aperture focusing technique in the frequency domain by using phased shift migration is robust against these perturbations. Further studies should be performed in order to assess this robustness feature as function of the flaw size and also its depth. Generalisation to composite structures should also be investigated.

\section{References}

1. A. Katunin, K. Dragan, M. Dziendzikowski, Composite Structures 127, 1 (2015)

2. S. Li, T.P. Chu, IEEE Transactions on Ultrasonics, Ferroelectrics, and Frequency Control 60, 2575 (2013)

3. C. Lane, the development of a $2 D$ ultrasonic array inspection for single crystal turbine blades (Springer International Publishing, Switzerland, 2014)

4. C.A. Wiley, IEEE Transactions on Aerospace and Electronic Systems AES-21, 440 (1985)

5. L. Ferro-Famil, E.Pottier, synthetic aperture radar imaging (Microwave Remote Sensing of Land Surface, Elsevier, 2016)

6. M.P. Hayes, P.T. Gough, IEE Journal of Oceanic Engineering 34, 207 (2009)

7. S.R. Doctor, T.E. Hall, L.D. Reid, NDT International 19, 163 (1986)

8. R.A. Kline, SAFT Imaging in Anisotropic Media (Review of Progress in QNDE, Plenum Press, New York, 1999)

9. M. Spies, W. Jager, Ultrasonics 41, 125 (2003)

10. M. Karaman, P.C. Li, IEEE Transactions on Ultrasonics, Ferroelectrics, and Frequency Control 42, 429 (1995)

11. Q. Li, X. Yang, P. Huang, Y. Liao, Journal of Theoretical and Applied Information Technology 47, 740 (2013)

12. T. Olofsson, IEEE Transactions on Ultrasonics, Ferroelectrics, and Frequency Control 57, 2522 (2010)

13. J. Gazdag, Geophysics 43, 1342 (1978) 